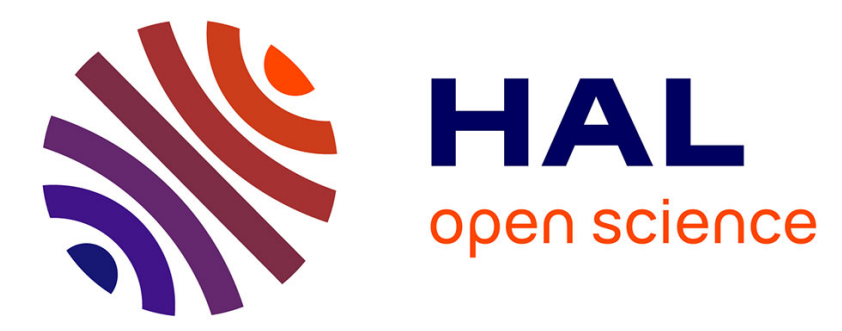

\title{
Empirically simulated spatial sorting points at fast epigenetic changes in dispersal behaviour
}

Katrien Hilde Petra van Petegem, Julien Pétillon, D Renault, Nicky Wybouw, Thomas van Leeuwen, Robby Stoks, Dries Bonte

\section{- To cite this version:}

Katrien Hilde Petra van Petegem, Julien Pétillon, D Renault, Nicky Wybouw, Thomas van Leeuwen, et al.. Empirically simulated spatial sorting points at fast epigenetic changes in dispersal behaviour. Evolutionary Ecology, 2015, 29 (2), pp.299-310. 10.1007/s10682-015-9756-9 . hal-01142665

HAL Id: hal-01142665

https://hal-univ-rennes1.archives-ouvertes.fr/hal-01142665

Submitted on 19 May 2015

HAL is a multi-disciplinary open access archive for the deposit and dissemination of scientific research documents, whether they are published or not. The documents may come from teaching and research institutions in France or abroad, or from public or private research centers.
L'archive ouverte pluridisciplinaire HAL, est destinée au dépôt et à la diffusion de documents scientifiques de niveau recherche, publiés ou non, émanant des établissements d'enseignement et de recherche français ou étrangers, des laboratoires publics ou privés. 


\section{Empirically simulated spatial sorting points at fast epigenetic}

\section{2 changes in dispersal behaviour}

3

4 Authors:

5 Katrien Hilde Petra Van Petegem ${ }^{1}$, Julien Pétillon ${ }^{1,2}$, David Renault ${ }^{3}$, Nicky Wybouw ${ }^{4,5}$,

6 Thomas Van Leeuwen ${ }^{5}$, Robby Stoks ${ }^{6}$, Dries Bonte ${ }^{1}$

7

8 Affiliations:

$9{ }^{1}$ Ghent University, Dep. Biology, Terrestrial Ecology Unit, K.L. Ledeganckstraat 35, B-9000 10 Ghent, Belgium

$11{ }^{2}$ Université de Rennes 1, EA 7316 Biodiversité et Gestion des Territoires, 263 Avenue du

12 Général Leclerc, CS 74205, 35042 Rennes Cedex, France

$13{ }^{3}$ Université de Rennes 1, UMR CNRS 6553 Ecobio, Campus de Beaulieu, 263 Avenue du 14 Général Leclerc, 35042 Rennes Cedex, France

$15{ }^{4}$ Ghent University, Dep. Crop Protection, Laboratory of Agrozoology, Coupure Links 653, B169000 Ghent, Belgium

$17{ }^{5}$ University of Amsterdam, Institute for Biodiversity and Ecosystem Dynamics (IBED), 18 Science Park 904, 1098 XH Amsterdam, The Netherlands

196 KU Leuven, Laboratory of Aquatic Ecology, Evolution and Conservation, Charles 20 Deberiotstraat 32, B-3000 Leuven, Belgium

Corresponding author:

23 Katrien Hilde Petra Van Petegem, E-mail: Katrien.VanPetegem@ugent.be

E-mail addresses of the co-authors: 
1 Julien.Petillon@univ-rennes1.fr; David.Renault@univ-rennes1.fr;

$2 \quad$ N.R.Wybouw@uva.nl; T.B.S.VanLeeuwen@uva.nl;

3 Robby.Stoks@bio.kuleuven.be; Dries.Bonte@ugent.be

4

5 Running title: Epigenetic inheritance of aerial dispersal

6

7 Keywords: range expansion, founder effect, Tetranychus urticae, artificial selection, maternal 8 inheritance, endosymbionts

9

Total word count: 3933

11 Word count per section: Abstract: 207, Introduction: 870, Materials and methods: 1152,

12 Results: 273, Discussion: 1334, Acknowledgements: 89

13

14 Number of cited references: 52

15 Number of figures and tables: figures: 3, tables: 1 


\section{Abstract}

2 species.

During range expansion, the most dispersive individuals make up the range front, and assortative mating between these dispersive phenotypes leads to increased dispersiveness (i.e. spatial sorting). The precise inheritance of dispersal, however, is to date largely unknown in many organisms, thereby hampering any progress in evaluating the adaptive potential of species during range expansion.

Using the spider mite Tetranychus urticae, we therefore empirically simulated spatial sorting by means of artificial selection on a unique pre-dispersal behaviour, tightly related to emigration. To separate directionality of the response from potential drift, we mimicked a recurrent low number of founders in replicated selection regimes. Afterwards, we inferred the mode of inheritance of the pre-dispersal behaviour by performing reciprocal crosses between selected (i.e. dispersive) and non-selected (i.e. non-dispersive) mites and by screening for endosymbionts known to be associated with changes in dispersal behaviour.

Despite the recurrent low number of founders, the aerial dispersal behaviour responded strongly to the imposed selection pressure. The behaviour furthermore showed a maternal inheritance, though independent of any known dispersal-related endosymbionts. Though cytoplasmic inheritance cannot fully be excluded, we attribute the observed strong and rapid, maternally influenced response in dispersal to transgenerational epigenetic effects. 


\section{Introduction}

2

Dispersal is the main mechanism maintaining gene flow among populations, and of primary importance for population regulation (Krebs et al. 1969), metapopulation dynamics (Hanski 1999; Hanski and Gaggiotti 2004; Levins 1969) and the spatial distribution of populations (Holt 1985; Kot et al. 1996). Crucially, dispersal is the key to the dynamics of genetic diversity in space and time (Gaggiotti and Couvet 2004), allowing species to respond to environmental challenges. Global warming, for example, is currently creating exciting evolutionary experiments where species expanding their ranges experience a strong selection for dispersal (Perkins et al. 2013; Shine et al. 2011). Indeed, during range expansion, the most dispersive individuals make up the range front, and assortative mating between these individuals (i.e. 'the Olympic Village effect') results in increased dispersal abilities (Phillips et al. 2010). This process is referred to as spatial sorting (or spatial selection) (Shine et al. 2011).

Range expansion is typically achieved by only those few individuals that reach and successfully settle in a previously unoccupied environment (i.e. not occupied by conspecifics). These individuals thus represent only a limited subset of the original genetic variation (i.e. founder effect) (Mayr 1963). Moreover, because of the low number of individuals, the new population will be highly susceptible to inbreeding and genetic drift, resulting in a clear genetic signature of range expansion, even in species with high dispersal capacity (Pierce et al. 2014). Repeated founder effects may furthermore result in gene surfing: a process where neutral alleles hitchhike with pioneer genotypes, thereby attaining high frequencies over large areas while not being under direct selection (Klopfstein et al. 2006). Life history-traits in founder populations will thus be shaped through the combined effect of 
natural selection (i.e. adaptation to the new local environment), spatial sorting (i.e. selection for dispersal and correlated traits) and genetic drift.

While selection on dispersal is expected to be strong during spatial sorting, the rate of evolutionary change will largely depend on the genetic architecture of the trait, the trait heritability and, equally, on the degree of adaptive plasticity potentially imposed by 'nonclassical' inheritance (see Moran and Alexander 2014 for a complete overview of limits to evolutionary responses to environmental change). The latter mechanism might be especially relevant in cases where standing genetic variation is low, like in case of subsequent founder effects during range expansion. Indeed, there is mounting evidence that parental effects and cytoplasmic inheritance might be alternative pathways for species to respond fast and adaptively to environmental perturbations (Bonduriansky et al. 2012; Bossdorf et al. 2008; Ledon-Rettig et al. 2013). Parental effects on dispersal, described for both plants (reviewed in Roach and Wulff 1987) and animals (e.g. Bitume et al. 2011; Diss et al. 1996; Li and Margolies 1994; Sinervo et al. 2006), as well as cytoplasmic inheritance of, for example, mitochondrial DNA (see Van Leeuwen et al. 2008) or endosymbiotic bacteria (see Goodacre et al. 2009), are therefore expected to induce fast phenotypic changes that might affect future ecological dynamics in a profound manner (but see Donohue 1999).

The two-spotted spider mite (Tetranychus urticae Koch - Acari, Tetranychidae) is a very useful model species for testing these dynamics, as previous research demonstrates a significant and high broad-sense genetic component underlying ambulatory $\left(\mathrm{h}^{2}=0.52\right.$ in Bitume et al. 2011) and aerial $\left(\mathrm{h}^{2}=0.22 \mathrm{in} \mathrm{Li}\right.$ and Margolies 1993) dispersal behaviour. These behaviours can easily be quantified under standardised laboratory conditions. The species is considered as one of the most damaging pest species and is expanding its natural range 
1

towards higher latitudes (see Carbonnelle et al. 2007). This range expansion is assumed to be due to long distance dispersal events through passive aerial dispersal, where new populations are founded by a limited number of individuals. In contrast, local dynamics are merely mediated by short distance ambulatory dispersal in response to elevated densities and kin competition (Bitume et al. 2013).

(1)

We simulated the spatial sorting process, as is expected to occur during range expansion, by means of artificial selection on a unique pre-dispersal behaviour, tightly related to emigration: the aerial take-off posture, which has a very high probability of resulting in mites becoming airborne (see Smitley and Kennedy 1985). We used T. urticae as a model species and mimicked a recurrent low number of founders in replicated selection regimes, to separate directionality of the response from potential drift. Several other studies already performed artificial selection on dispersal in T. urticae, but these focused on ambulatory dispersal (Bitume et al. 2011; Tien et al. 2011; Yano and Takafuji 2002) or used a very high number of individuals ( $\mathrm{Li}$ and Margolies 1994) and can therefore not be interpreted in the context of spatial sorting during range expansion.

Our aims were twofold. Firstly, we assessed the evolutionary response of the aerial take-off behaviour to a strong selection pressure, given only minimal genetic variation. Secondly, we assessed the mode of inheritance of the aerial dispersal trait by crossing dispersers and non-dispersers and by screening for five endosymbiotic bacteria known to be associated with changes in dispersal behaviour (Goodacre et al. 2009). We hypothesised that a fast evolutionary response in aerial dispersal behaviour in $T$. urticae could be the result of 'non-classical' inheritance, as indicated by the slight maternal influence in the study of Li and Margolies (1994). 


\section{Materials and methods}

2

\section{$3 \quad$ Study species}

\section{Artificial selection}

The two-spotted spider mites (Tetranychus urticae Koch - Acari, Tetranychidae) used in this study originated from a laboratory strain ("LS-VL") that was originally collected in October 2000 from roses in a garden near Ghent, Belgium. Since then, this strain was maintained on potted beans (Phaseolus vulgaris L. cv. 'Prelude') in a climate-controlled room at $26 \pm 0.5^{\circ} \mathrm{C}, 60 \% \mathrm{RH}$ and $16 / 8 \mathrm{~h}(\mathrm{~L} / \mathrm{D})$ photoperiod (Van Leeuwen et al. 2006).

We largely based our experimental procedure on Li and Margolies (1993, 1994). The propensity to disperse by air was assessed by counting the percentage of female mites showing the aerial take-off posture (i.e. upraised first pair of legs and cephalothorax, to increase drag). Dispersal latency was furthermore assessed by counting the number of minutes between the start of the one hour observation and the moment the focal female showed the aerial take-off posture. Only one-day-old, freshly mated females were considered, since they are the main dispersing stage (see Li and Margolies 1993, 1994 for more details).

To ensure having a sufficient number of dispersive individuals to start the selection experiment (dispersal propensity was relatively low in the base population), an initial selection procedure was first run. For this initial generation of selection, 20 females were put on each of three separate arenas -black painted squares of hard plastic on wet cotton. They were then subjected to a light and wind regime known to elicit dispersal initiation behaviour (i.e. the aerial take-off posture) in T. urticae (see Li and Margolies 1993). During one hour, the females were observed and screened for the aerial take-off posture in a climate room at $20^{\circ} \mathrm{C}$. From one of the three arenas, the first five females showing the aerial take-off posture 
1

were collected and put separately on a fresh bean leaf. These would make up the high dispersal regime (HD). On a second arena, all females showing the posture were killed, and afterwards, five females were randomly chosen from the remaining ones. These females were then put on a fresh bean leaf and made up the low dispersal regime (LD). For the control regime (C), five females were randomly chosen out of all original 20 females from the third arena and put on yet another fresh bean leaf. All of these females (HD, LD, C) were subsequently allowed to lay eggs in a breeding room at $20^{\circ} \mathrm{C}$ with a light-regime of 16:8 LD. Their female offspring, once adult and freshly mated, were then used for the next generation of selection.

From this point onwards, the selection experiment was performed, using three replicates per selection regime (thus nine lines, hence nine arenas to be observed: HD1, HD2, HD3, LD1, LD2, LD3, C1, C2, C3). Using these replicates allowed us to differentiate between random genetic drift and evolutionary changes caused by the selective pressure. The selection criteria remained the same as for the initial selection procedure (see above). In cases where less than five females showed the aerial take-off posture in a HD line, we just continued with this lower number (i.e. we did not increase the number to five by adding randomly chosen females as this would weaken the selection pressure).

In order to create sufficient divergence between the selection regimes, the selection procedure was repeated for 13 generations (Li and Margolies 1994). Dispersal latency was only assessed for the HD regime, as too few females showed the dispersal behaviour in the $\mathrm{C}$ and LD regime.

\section{Crosses between selection regimes}

The adult HD and LD females resulting from the 13th generation of selection were put on fresh bean leaves and allowed to lay eggs. The freshly adult male and female offspring of 
1 the six lines ( 2 selection regimes $x 3$ replicates) were then used for the crosses. This

2 experiment consisted of females and males from HD and LD selection regimes mating

3 amongst each other according to a $2 \times 2$-factorial design. (To ensure a sufficient number of

4 female offspring to later conduct an aerial dispersal test, five females were each time put

5 together with five males and allowed to mate for four days.) The crosses ( $\mathrm{HD}_{\mathrm{fem}} \mathrm{X} \mathrm{HD}_{\text {male }}$,

$6 \mathrm{HD}_{\text {fem }} \mathrm{x} \mathrm{LD}_{\text {male}}, \mathrm{LD}_{\text {fem }} \mathrm{x} \mathrm{HD}_{\text {male}}, \mathrm{LD}_{\text {fem }} \mathrm{x} \mathrm{LD}_{\text {male }}$ ) were made in each of the three original

7 replicates of the HD and LD selection regimes, resulting in a total of twelve $(3 \times 4)$ crosses.

8 Finally, the one-day-old female offspring from these crosses were all tested for their aerial

9 dispersal propensity. This was done in a similar setup as described earlier. Twelve $(3 \times 4)$

10 arenas were observed for one hour, during which all females showing the aerial take-off

11 posture were immediately removed from their arena. Such a removal was each time recorded.

12 As such, the proportion of females showing the posture could afterwards be calculated for

13 each arena by dividing the number of removals by the original number of females on that 14 arena.

\section{Endosymbionts}

Four hundred adult females were pooled from the parental spider mite strain prior to selection and from the HD, LD and C selection regimes after the 13th generation of selection.

Genomic DNA was collected following a phenol-chloroform extraction method (Van

Leeuwen et al. 2008). Additionally, 20 DNA samples, each originating from a single adult

female mite, were extracted from the parental strain and from the selection regimes, following the procedure described in Van Leeuwen et al. (2008). In all DNA samples, the presence of five endosymbionts known to be associated with changes in dispersal behaviour (Cardinium, 
1 PCR, using the primers listed in Table 1. Amplified PCR products were sequenced by LGC

2 Genomics (Berlin, Germany) after purification with Cycle-Pure Kit (EZNA TM).

3

4

\section{Statistics}

All analyses were performed with SAS 9.4 (SAS Institute Inc. 2013), using the glimmix procedure for generalised linear mixed models (GLMM). We analysed aerial dispersal propensity with selection regime (HD, LD, C), generation and the interaction between selection regime and generation as the independent variables. For the analysis of aerial dispersal latency, only generation was used as an independent variable, as only the HD regime was involved. The analysis of the regime crosses was performed with the dispersal type of the mother and the father (i.e. originating from the HD selection regime opposed to the LD selection regime) and their interaction term as the independent variables. According to the dependent variable, a binomial (dispersal propensity) or Poisson (dispersal latency) error structure was modelled with the proper link function (logit/log). Replicate was always modeled as a random effect. This was done to control for dependency among the replicates of each selection regime. By modeling residual variation as an additional random factor, we corrected for potential overdispersion (Verbeke and Molenberghs 2000). The denominator degrees of freedom for the tests of fixed effects were computed according to a general Satterthwaite approximation. All non-significant contributions $(p>0.05)$ were omitted by a backwards selection procedure. Finally, post-hoc Tukey tests were used to obtain the pairwise differences among treatments (only for the regime crosses). 


\section{Results}

2

3

\section{Artificial selection}

Aerial dispersal propensity responded well to the artificial selection: selection regime significantly interacted with generation $\left(\mathrm{F}_{2,1673}=4.72 ; \mathrm{p}=0.0091\right)$ (Fig. 1). The slopes of the $\mathrm{HD}$ and $\mathrm{LD}$ regime differed significantly from zero: $\mathrm{HD}: 0.0696 \pm 0.0210\left(\mathrm{t}_{1835}=3.32\right.$; $\mathrm{p}=0.0009), \mathrm{LD}:-0.1024 \pm 0.0370\left(\mathrm{t}_{1835}=-2.77 ; \mathrm{p}=0.0056\right), \mathrm{C}:-0.0509 \pm 0.0307\left(\mathrm{t}_{1835}=-1.66\right.$; $\mathrm{p}=0.0977$ ). These slopes imply that the odds of successful dispersal multiply by a factor of 1.07, 0.90 and 0.95 per generation, in the HD, LD and C regime, respectively.

Furthermore, latency (which was only measured for the HD regime) significantly decreased with generation $\left(\mathrm{F}_{1,215}=112.81 ; \mathrm{p}<0.0001\right)$ (Fig. 2).

\section{Crosses between selection regimes}

The proportion of female offspring displaying the aerial take-off posture differed significantly among some of the crosses (Fig.3). Only the dispersal type of the mother had a significant effect $\left(\mathrm{F}_{1,8}=19.40 ; \mathrm{p}=0.0023\right)$ : the proportion of females showing the aerial takeoff posture was higher for HD mothers $(0.4054 \pm 0.0496 \mathrm{SE})$ than for LD mothers $(0.0636 \pm$ $0.0287 \mathrm{SE})\left(\mathrm{t}_{8}=4.40 ; \mathrm{p}=0.0023\right)$. The dispersal type of the father did not affect the dispersal propensity of the offspring $\left(\mathrm{F}_{1,8}=2.44 ; \mathrm{p}=0.1566\right)$. There was also no significant interaction between the dispersal type of the mother and the father $\left(\mathrm{F}_{1,8}=0.37 ; \mathrm{p}=0.5587\right)$.

\section{Endosymbionts}

Wolbachia and Rickettsia were detected by species-specific primers in the pooled sample of the non-selected parental strain, and infection showed a prevalence of 0.15 and 0.9 respectively, as determined by single-mite PCR. BLASTn-searches, using the sequenced PCR 
1 products as queries against the NCBI database, confirmed the correct identification of

2 endosymbionts. No endosymbionts were found in the replicated HD, C and LD selection 3 regimes in both the pooled samples of 400 mites and the single mite DNA samples. 


\section{Discussion}

2

3

4

We empirically simulated the process of spatial sorting by artificially selecting on aerial dispersal behaviour (more specifically, the aerial take-off posture), using a recurrent low number of founders, and demonstrate a fast response to selection and a maternal inheritance of this dispersal behaviour in T. urticae.

(1)

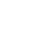

'highly dispersive mites' (HD selection regime) were selected for showing a specific behaviour, while 'low dispersive mites' (LD selection regime) were selected for not showing this behaviour. Possibly, the mites from the LD regime might therefore not have been selected for a low propensity to disperse, but for a general low viability, hence incapability of showing the aerial take-off posture (see Tien et al. 2011). However, even with our very strong selection criteria (the number of founders was each time restricted to only five mites), the aerial dispersal behaviour never really disappeared in the LD regime. This suggests that the capability for the aerial take-off posture remained present throughout the selection procedure. All mites were moreover maintained at similar, low densities, resulting in overall fit females in all selection treatments; i.e. the selection regimes did not differ in daily fecundity or longevity (Pétillon et al., unpublished data).

Despite the recurrent low number of founders used in our artificial selection experiment, the aerial dispersal behaviour responded equally strong to the imposed selection pressure as in the study of Li and Margolies (1994). Aerial dispersal propensity gradually increased in the HD regime while it showed a steady decrease in the LD regime. The strong response across all replicates, in a minimal number of generations, is unlikely to have resulted 
1 from new mutations or from standing genetic variation. Standing genetic variation was most

2 probably low due to the low number of initial founders that were used to start the replicated

3 selection regimes. Instead, in accordance with a growing amount of studies in lizards (e.g.

4 Massot et al. 2002; Meylan et al. 2002; Sinervo et al. 2006), birds (e.g. Tschirren et al. 2007)

5 and arthropods (e.g. Bitume et al. 2011, 2014; Bonte et al. 2007; Li and Margolies 1994;

6 Mestre and Bonte 2012), we attribute the strong response in our experiment to

7 intergenerational plasticity of dispersal through a maternal influence on offspring phenotype.

8 Indeed, the crosses between our selection regimes indicated a strong maternal inheritance.

9 Several mechanisms could be responsible for this, including cytoplasmic inheritance, classical

10 Mendelian inheritance through sex specific chromosomes and maternal effects.

Cytoplasmic inheritance includes, amongst others, the vertical transmission of bacterial endosymbionts. Goodacre et al. (2009) demonstrated an effect of such cytoplasmic endosymbionts on the tendency for long-distance movement in the spider Erigone atra. We thus investigated whether the maternal inheritance in our study could have been mediated by such maternally transmitted endosymbiotic bacteria. The absence of all endosymbionts known to be associated with changes in dispersal behaviour (Cardinium, Caulobacter, Rickettsia, Spiroplasma and Wolbachia) in our selection regimes, however, makes this mechanism unlikely. Surprisingly, where Rickettsia was absent in the selection regimes, it showed a high prevalence in the non-selected parental stock population. This apparent discrepancy may be ascribed to the density of Rickettsia bacteria within individual mites of the parental population. Although we measured the prevalence of endosymbionts in the mite populations, 23 we did not evaluate infection density (i.e. the number of Rickettsia within each single 24 individual). It is well established that this infection density affects many aspects of the 25 endosymbiont-host interaction, including the vertical transmission of these endosymbionts 
1 (Noda et al. 2001; Perrot-Minnot and Werren 1999). Low infection densities can cause

2 imperfect vertical transmission, and over multiple generations, this can lead to an eventual

3 overall loss of the endosymbiont. Our results suggest that the Rickettsia bacteria suffered from

4 imperfect vertical transmission, potentially caused by low bacterial densities within single

5 mites. Rickettsia can therefore not have been responsible for the maternal inheritance of aerial

6 dispersal behaviour in our experiment. Nonetheless, there could always be some other

7 endosymbiotic bacteria contributing to this observation.

Another form of cytoplasmic inheritance is the vertical transmission of mitochondrial

9 DNA. When individuals possess more than one mtDNA haplotype (i.e. mitochondrial heteroplasmy), genotype frequencies can shift greatly in a single transmission from mother to offspring, as for instance found for loci related to pesticide resistance (Van Leeuwen et al. 2008). Though mitochondrial heteroplasmy is generally rare (Jenuth et al. 1996), we should thus keep in mind that loci on the mitochondrial genome may also contribute to strong and rapid phenotypic effects.

Maternal inheritance of a trait can in some cases also be the consequence of classical Mendelian inheritance of the mother's nuclear genome. If dispersal-related traits are controlled by loci on the female sex-chromosome (as is, for example, present in butterflies, where females are the heterogametic sex), Mendelian inheritance could be enough to explain mother to daughter similarities. This, however, does not apply for T. urticae. In tetranychid species, sexual reproduction occurs through arrhenotokous parthenogenesis, in which unfertilised eggs develop into males and fertilised eggs into females. T. urticae females do not possess a 'female-specific chromosome' that is passed on from mother to daughter only. Mendelian inheritance of nuclear genomes can therefore not explain the result of our crossmating experiment. 
A third potential mechanism causing the observed maternal inheritance could be maternal effects. Maternal effects are defined as any maternal influence on offspring phenotype that cannot be attributed solely to offspring genotype, the direct action of the non-

5 maternal components of the offspring environment, or to their combination (Lacey 1998). 6 According to Lacey (1998), there are three types: (i) genetically based maternal effects (e.g. 7 genomic imprinting), (ii) indirect genetic maternal effects (e.g. genetically determined maternal care) and (iii) environmentally induced maternal effects (i.e. all effects induced by

9 the maternal environment, independent of maternal genotype). Since T. urticae does not provide any form of maternal care and since all individuals were raised in identical circumstances for several generations, we can rule out indirect genetic and environmentally induced maternal effects. Though we did not specifically assess any genetically based maternal effect, we consider it most likely that such transgenerational epigenetic effects caused the maternal inheritance of aerial dispersal behaviour observed in our experiment.

In the context of the current global change, the potential of species to colonise new habitats may be essential for their persistence. Since shifts at the leading edge of expanding ranges are often achieved by a small number of long-distance dispersing individuals, newly established populations may suffer from founder bottlenecks (reviewed in Hill et al. 2011). Not only does this lead to a reduced genetic diversity, with all its possible consequences, it also implies that the individuals at expanding ranges may differ substantially from the ones that stay behind. Individuals at the shifting edge may carry a suite of typical characteristics, shaped through selection on dispersal and other potentially correlated traits that may eventually constrain genetic adaptation. In the presence of such genetic constraints, transgenerational epigenetic inheritance may provide alternative pathways for species to 
1 respond fast and adaptively to environmental perturbations like global change (Bossdorf et al.

2 2008; Ledon-Rettig et al. 2013; Tuomainen and Candolin 2011). The ability to epigenetically

3 control dispersal can be considered as a form of phenotypic plasticity that evolved in response

4 to the ephemeral nature of the species' habitat ( $T$. urticae is characterised by a rapid

5 development and a high fecundity, leading to exponential growth and systematic depletion of

6 its host plant). Indeed, spatiotemporal variation in habitat quality is known to select for

7 plasticity as an adaptive trait on its own (Via and Lande 1985).

8

In conclusion, despite the recurrent low number of founders used to simulate the process of spatial sorting during range expansion, the aerial dispersal behaviour in T. urticae responded strongly to the imposed artificial selection pressure. We attribute this strong and rapid response to transgenerational epigenetic mechanisms, though we should acknowledge that cytoplasmic inheritance cannot fully be excluded as a potential (co-)affecting factor. As a consequence of this epigenetic inheritance, we can expect fast, non-classical evolutionary dynamics during range expansion in the species. 


\section{Acknowledgements}

2

3 This project was funded by the Fund for Scientific Research - Flanders (FWO)

4 (project G.0610.11). We also thank the Fund for Scientific Research - Flanders (FWO) for

5 funding JP (visiting postdoctoral fellowship: FWO-project G. G0057/09N). DB and RS were

6 supported by BelSpo IAP Project "Speedy". We furthermore thank the INEE-CNRS

7 (ENVIROMICS call, project 'ALIENS') for funding DR. Finally, we thank Pim Edelaar,

8 Cristina García and John Endler for inviting us and we thank two anonymous reviewers and

9 Pim Edelaar for their useful comments on earlier versions of this manuscript. 


\section{$1 \quad$ References}

2

3 Bitume EV, Bonte D, Magalhaes S et al (2011) Heritability and Artificial Selection on

4 Ambulatory Dispersal Distance in Tetranychus urticae: Effects of Density and Maternal

5 Effects. Plos One 6(10)

6 Bitume EV, Bonte D, Ronce O et al (2013) Density and genetic relatedness increase dispersal

7 distance in a subsocial organism. Ecology Letters 16(4):430-437

8 Bitume EV, Bonte D, Ronce O et al (2014) Dispersal distance is influenced by parental and 9 grand-parental density. Proceedings of the Royal Society B-Biological Sciences 281(1790) Bonduriansky R, Crean AJ, Day T (2012) The implications of nongenetic inheritance for evolution in changing environments. Evolutionary Applications 5(2):192-201

Bonte D, Van Belle S, Maelfait JP (2007) Maternal care and reproductive state-dependent mobility determine natal dispersal in a wolf spider. Animal Behaviour 74:63-69

Bossdorf O, Richards CL, Pigliucci M (2008) Epigenetics for ecologists. Ecology Letters 11(2):106-115

Carbonnelle S, Hance T, Migeon A et al (2007) Microsatellite markers reveal spatial genetic structure of Tetranychus urticae (Acari : Tetranychidae) populations along a latitudinal gradient in Europe. Experimental and Applied Acarology 41(4):225-241

Diss AL, Kunkel JG, Montgomery ME et al (1996) Effects of maternal nutrition and egg provisioning on parameters of larval hatch, survival and dispersal in the gypsy moth, Lymantria dispar L. Oecologia 106(4):470-477

Donohue K (1999) Seed dispersal as a maternally influenced character: Mechanistic basis of maternal effects and selection on maternal characters in an annual plant. American Naturalist 154(6):674-689 
1 Fukatsu T, Nikoh N (2000) Endosymbiotic microbiota of the bamboo pseudococcid Antonina

2 crawii (Insecta, Homoptera). Applied and Environmental Microbiology 66(2):643-650

3 Gaggiotti OE, Couvet D (2004) Genetic structure in Heterogeneous environments. In: Ferriere

4 R., Dieckmann U., Couvet D. (eds) Evolutionary Conservation Biology. Cambridge

$5 \quad$ University Press, Cambridge, pp 229-243

6 Goodacre SL, Martin OY, Bonte D et al (2009) Microbial modification of host long-distance

7 dispersal capacity. BMC Biology 7:32

8 Gotoh T, Noda H, Ito S (2007) Cardinium symbionts cause cytoplasmic incompatibility in

$9 \quad$ spider mites. Heredity 98(1):13-20

Hanski I (ed) (1999) Metapopulation Ecology. Oxford University Press, Oxford

Hanski I, Gaggiotti OE (eds) (2004) Ecology, Genetics and Evolution of Metapopulations. Elsevier Academic Press, San Diego

Hill JK, Griffiths HM, Thomas CD (2011) Climate change and evolutionary adaptations at species' range margins. Annual Review of Entomology 56:143-159

Holt RD (1985) Population dynamics in two-patch environments: some anomalous consequences of an optimal habitat distribution. Theoretical Population Biology 28(2):181208

Hoy MA, Jeyaprakash A (2005) Microbial diversity in the predatory mite Metaseiulus occidentalis (Acari : Phytoseiidae) and its prey, Tetranychus urticae (Acari : Tetranychidae). Biological Control 32(3):427-441

Jenuth JP, Peterson AC, Fu K et al (1996) Random genetic drift in the female germline explains the rapid segregation of mammalian mitochondrial DNA. Nature Genetics 14(2):146151 
1 Jeyaprakash A, Hoy MA (2000) Long PCR improves Wolbachia DNA amplification: wsp

2 sequences found in 76\% of sixty-three arthropod species. Insect Molecular Biology 9(4):393-

3405

4 Klopfstein S, Currat M, Excoffier L (2006) The fate of mutations surfing on the wave of a

5 range expansion. Molecular Biology and Evolution 23(3):482-490

6 Kot M, Lewis MA, van den Driessche P (1996) Dispersal data and the spread of invading

7 organisms. Ecology 77(7):2027-2042

8 Krebs CJ, Keller BL, Tamarin RH (1969) Microtus population biology - demographic

9 changes in fluctuating populations of M. ochrogaster and M. pennsylvanicus in Southern

10 Indiana. Ecology 50(4):587-607

11 Lacey EP (1998) What is an adaptive environmentally induced parental effect? In: Mousseau

12 TA and Fox CW (eds) Maternal effects as adaptations. Oxford University Press, New York, 13 pp 54-66

14 Ledon-Rettig CC, Richards CL, Martin LB (2013) Epigenetics for behavioral ecologists.

15 Behavioral Ecology 24(2):311-324

16 Levins R (1969) Some demographic and genetic consequences of environmental 17 heterogeneity for biological control. Bulletin of the Entomological Society of America $18 \quad 15: 237-240$

19 Li JB, Margolies DC (1993) Quantitative genetics of aerial dispersal behavior and life-history 20 traits in Tetranychus urticae. Heredity 70:544-552

21 Li JB, Margolies DC (1994) Responses to direct and indirect selection on aerial dispersal 22 behavior in Tetranychus urticae. Heredity 72:10-22

23 Massot M, Clobert J, Lorenzon P et al (2002) Condition-dependent dispersal and ontogeny of 24 the dispersal behaviour: an experimental approach. Journal of Animal Ecology 71(2):253-261

25 Mayr E (1963) Animal species and evolution. Harvard University Press, Cambridge 
1 Mestre L, Bonte D (2012) Food stress during juvenile and maternal development shapes natal

2 and breeding dispersal in a spider. Behavioral Ecology 23(4):759-764

3 Meylan S, Belliure J, Clobert J et al (2002) Stress and body condition as prenatal and

4 postnatal determinants of dispersal in the common lizard (Lacerta vivipara). Hormones and

5 Behavior 42(3):319-326

6 Moran EV, Alexander JM (2014) Evolutionary responses to global change: lessons from

7 invasive species. Ecology Letters 17(5):637-649

8 Noda H, Koizumi Y, Zhang Q et al (2001) Infection density of Wolbachia and incompatibility

9 level in two planthopper species, Laodelphax striatellus and Sogatella furcifera. Insect

10 Biochemistry and Molecular Biology 31(6-7):727-737

11 Perkins AT, Phillips BL, Baskett ML et al (2013) Evolution of dispersal and life history 12 interact to drive accelerating spread of an invasive species. Ecology Letters 16(8):1079-1087

13 Perrot-Minnot MJ, Werren JH (1999) Wolbachia infection and incompatibility dynamics in experimental selection lines. Journal of Evolutionary Biology 12(2):272-282

Phillips BL, Brown GP, Shine R (2010) Life-history evolution in range-shifting populations.

Ecology 91(6):1617-1627

17 Pierce AA, Zalucki MP, Bangura M et al (2014) Serial founder effects and genetic differentiation during worldwide range expansion of monarch butterflies. Proceedings of the Royal Society B: Biological Sciences 281(1797)

Roach DA, Wulff RD (1987) Maternal Effects in Plants. Annual Review of Ecology and Systematics 18:209-235

22 Shine R, Brown GP, Phillips BL (2011) An evolutionary process that assembles phenotypes 23 through space rather than through time. Proceedings of the National Academy of Sciences of the United States of America 108(14):5708-5711 
1 Sinervo B, Calsbeek R, Comendant $\mathrm{T}$ et al (2006) Genetic and maternal determinants of

2 effective dispersal: The effect of sire genotype and size at birth in side-blotched lizards.

3 American Naturalist 168(1):88-99

4 Smitley DR, Kennedy GG (1985) Photo-oriented aerial dispersal behavior of Tetranychus

5 urticae (Acari, Tetranychidae) enhances escape from the leaf surface. Annals of the

6 Entomological Society of America 78(5):609-614

7 Tien NSH, Sabelis MW, Egas M (2011) Ambulatory dispersal in Tetranychus urticae: an 8 artificial selection experiment on propensity to disperse yields no response. Experimental and 9 Applied Acarology 53(4):349-360

Tschirren B, Fitze PS, Richner H (2007) Maternal modulation of natal dispersal in a passerine bird: An adaptive strategy to cope with parasitism? American Naturalist 169(1):87-93

12 Tuomainen U, Candolin U (2011) Behavioural responses to human-induced environmental change. Biological Reviews 86(3):640-657

Van Leeuwen T, Tirry L, Nauen R (2006) Complete maternal inheritance of bifenazate resistance in Tetranychus urticae Koch (Acari : Tetranychidae) and its implications in mode of action considerations. Insect Biochemistry and Molecular Biology 36(11):869-877 Van Leeuwen T, Vanholme B, Van Pottelberge S et al (2008) Mitochondrial heteroplasmy and the evolution of insecticide resistance: Non-Mendelian inheritance in action. Proceedings of the National Academy of Sciences of the United States of America 105(16):5980-5985 Verbeke G, Molenberghs G (eds) (2000) Linear mixed models for longitudinal data. SpringerVerlag New York Inc., New York Via S, Lande R (1985) Genotype-environment interaction and the evolution of phenotypic plasticity. Evolution 39(3):505-522 
1 Weeks AR, Velten R, Stouthamer R (2003) Incidence of a new sex-ratio-distorting

2 endosymbiotic bacterium among arthropods. Proceedings of the Royal Society B-Biological

3 Sciences 270(1526):1857-1865

4 Weisburg WG, Barns SM, Pelletier DA et al (1991) 16S ribosomal DNA amplification for 5 phylogenetic study. Journal of Bacteriology 173(2):697-703

6 Yano S, Takafuji A (2002) Variation in the life history pattern of Tetranychus urticae (Acari :

7 Tetranychidae) after selection for dispersal. Experimental and Applied Acarology 27(1-2):1$8 \quad 10$

9 


\section{Figure legends}

2

3 Figure 1: Evolution of aerial dispersal propensity. For each generation, the mean aerial male/female.

Figure 2: Evolution of aerial dispersal latency. For each generation, the mean aerial dispersal latency in the high dispersal regime (HD) is represented by a dot. Standard errors are represented by bars. Aerial dispersal latency is averaged over replicates and females within a replicate. The aerial dispersal latency of a female mite is the number of minutes between the start of the one hour observation of the aerial dispersal propensity of this female and the moment she shows the aerial take-off posture. (Note: Due to circumstances, the selection procedure did not last a full hour in generation 8.)

Figure 3: Aerial dispersal propensity in the offspring of the different regime crosses. The mean aerial dispersal propensity of the female offspring from the four different regime crosses is represented by columns. Standard errors are represented by bars. Different letters above the error bars indicate significantly different outcomes according to the used statistical test. Aerial dispersal propensity is averaged over the replicates within each of the regime crosses and over female offspring within a replicate. $\mathrm{HD}_{\mathrm{m} / \mathrm{f}}$ : high dispersal male/female, $\mathrm{LD}_{\mathrm{m} / \mathrm{f}}$ : low dispersal

25 


\section{Figures}

2

$3 \quad$ Figure 1

aerial dispersal propensity

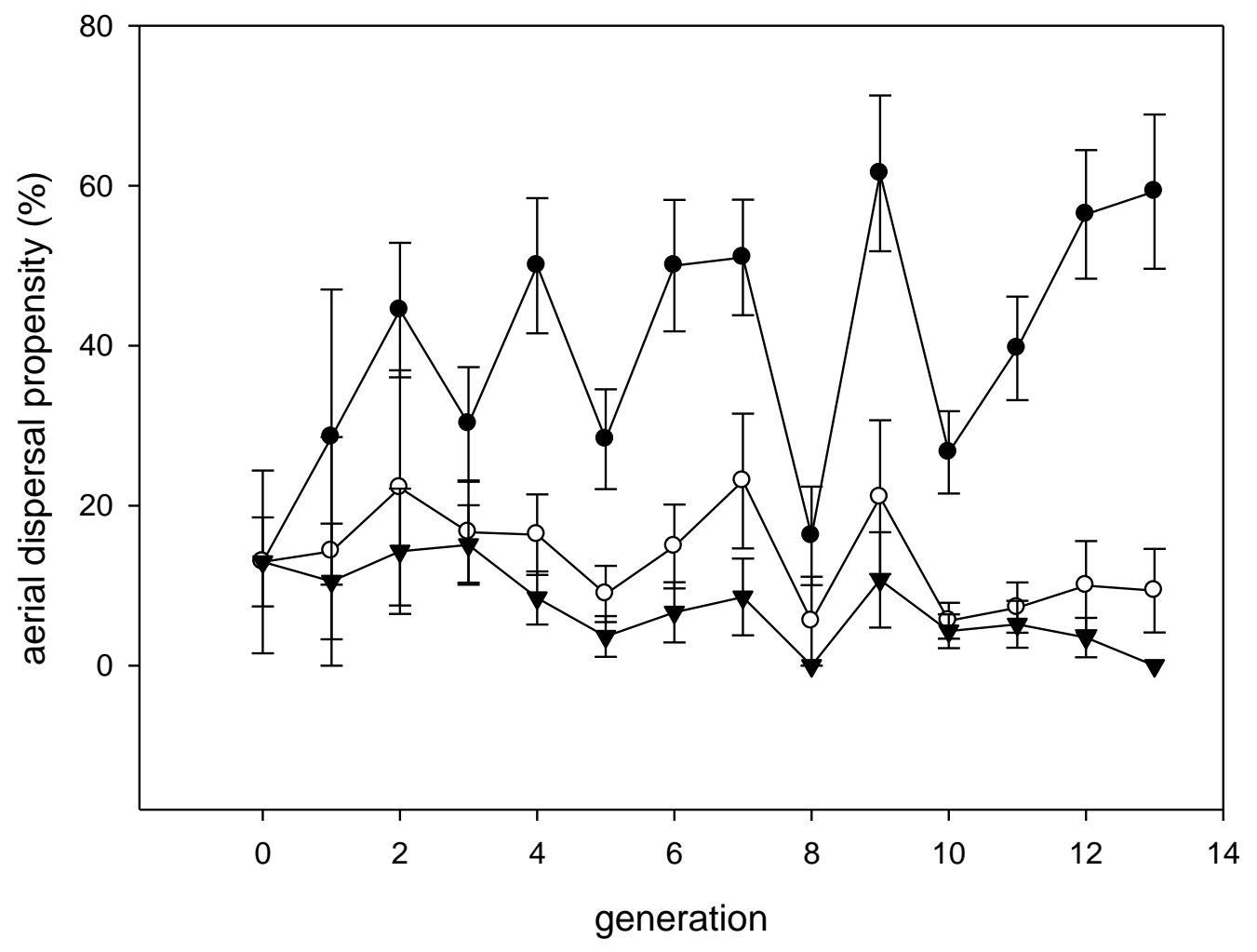

5 


\section{$1 \quad$ Figure 2}

aerial dispersal latency

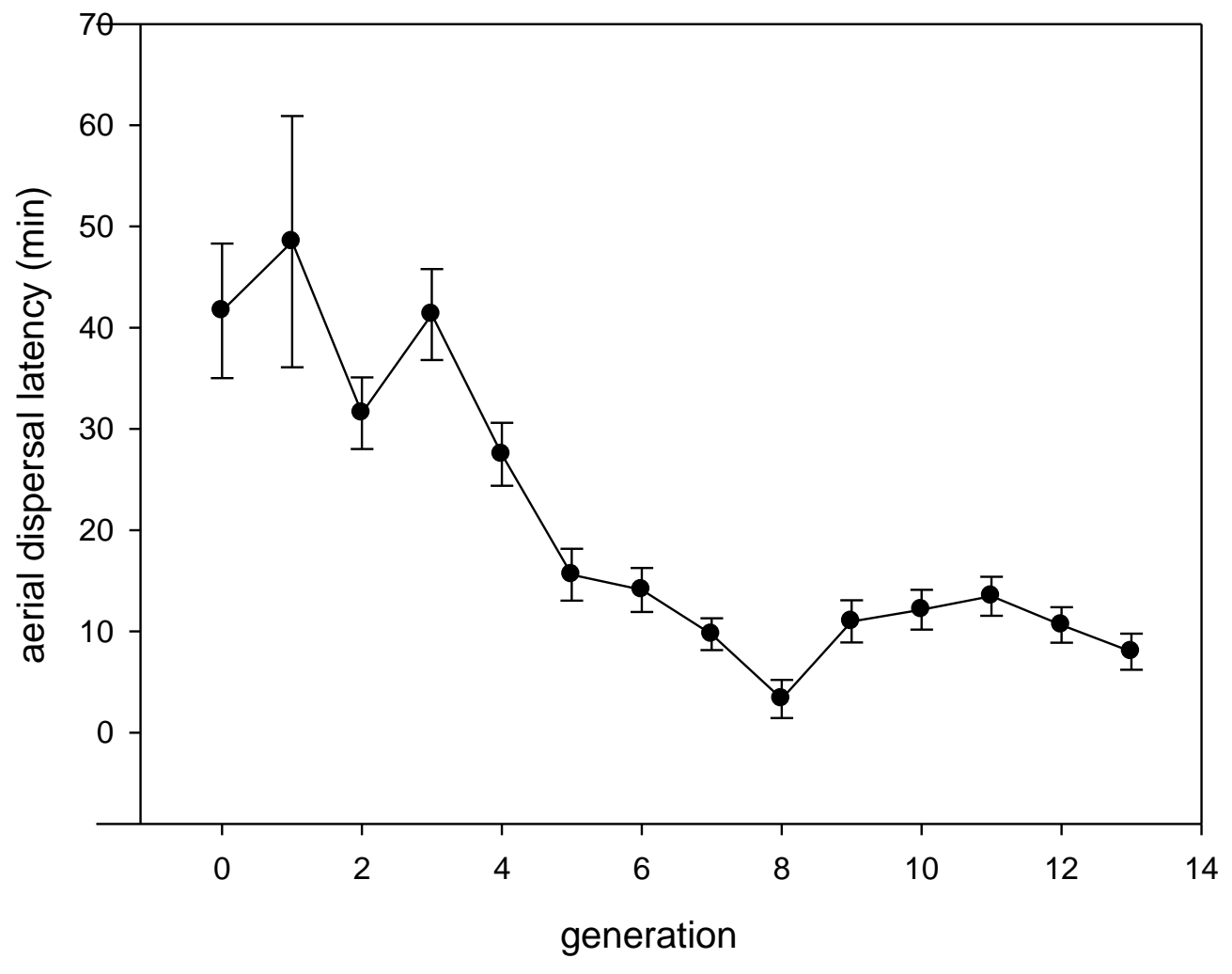

3 
$1 \quad$ Figure 3

inheritance of aerial dispersal

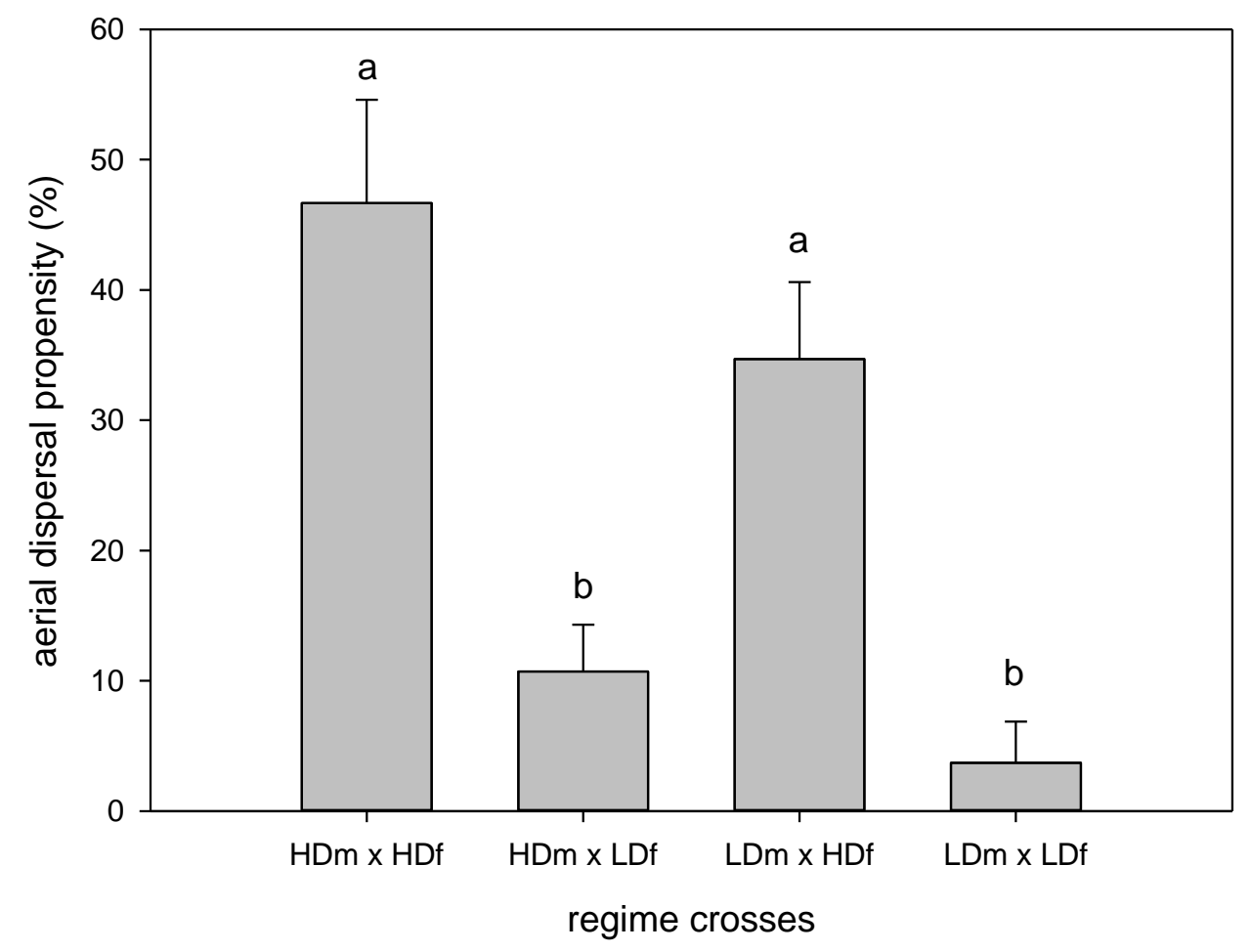

3 


\section{Tables}

2

$3 \quad$ Table 1

4 Primers used in this study for the detection of endosymbionts in T. urticae. For each primer pair, sequences are given for the forward (F) and the

5 reverse (R) primer, with the corresponding reference. The presence of Cardinium was investigated using three different primer pairs.

6 Bacteria Primer sequence [5' - 3'] Reference

7 Cardinium F1: GCGGTGTAAAATGACGTG (Weeks et al. 2003)

8 R1: ACCTMTTCTTAACTCAAGCCT

9

F2: GGAACCTTACCTGGGCTAGAATGTATT

(Gotoh et al. 2007)

R2: GCCACTGTCTTCAAGCTC TACCAA

12

R3: TCTGTGCCCATGGTTACGCC 
R: CTTGCGAGTTAGCGCAACG

2 Wolbachia F: TGGTCCAATAAGTGATGAAGAAACTAGCTA (Jeyaprakash and Hoy 2000)

R: AAAAATTAAACGCTACTCCAGCTCTGCAC

F: GGACTAATTGGGGTTTGCTC

(Hoy and Jeyaprakash 2005)

R: GCTGCCTCTTGCGTTAGCT

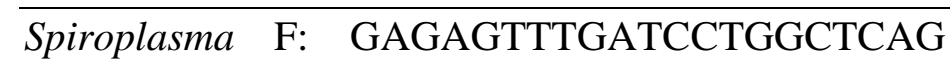

8 